\title{
ON THE LOCAL STRUCTURE OF QUANTIZATIONS IN CHARACTERISTIC $p$
}

\author{
AKAKI TIKARADZE
}

\begin{abstract}
Let $A$ be a central quantization of an affine Poisson variety $X$ over a field of characteristic $p>0$. We show that the completion of $A$ with repect to a closed point $y \in X$ is isomorphic to the tensor product of the Weyl algebra with a local Poisson algebra. This result can be thought of as a positive characteristic analogue of results of Losev and Kaledin about slice algebras of quantizations in characteristic 0.
\end{abstract}

\section{Dedicated to my mother Natalia Tikaradze}

\section{INTRODUCTION}

Let $\mathbf{k}$ be an algebraically closed field of characteristic $p>2$. By a Poisson algebra over $\mathbf{k}$ we will understand a commutative $\mathbf{k}$-algebra equipped with a k-linear Poisson bracket $\{$,$\} . Throughout given a Poisson algebra B$ and $b \in B$, by $\operatorname{ad}(b)$ we will denote the corresponding derivation $\{b$,$\} .$ While for a non-commutative algebra $A$ and its element $a \in A$, by $\operatorname{ad}(a)$ we will denote the commutator $[a, \quad]$. Also, for an algebra $A$ by $Z(A)$ we will denote its center. By a (deformation) quantization of a Poisson algebra $(B,\{\}$,$) we will understand an associative \mathbf{k}[[\hbar]]$-algebra $A$ such that $A$ is topologically free $\mathbf{k}[[\hbar]]$-module, $B=A / \hbar A$, and

$$
\{a \bmod \hbar, b \bmod \hbar\}=\left(\frac{1}{\hbar}[a, b]\right) \quad \bmod \hbar, \quad a, b \in A .
$$

Motivated by the notion of central quantizations introduced by Bezrukavnikov and Kaledin [[BK], Definition 1.2], we will give the following

Definition 1.1. A quantization $A$ of a Poisson k-algebra $B$ is called weakly central if for any $a \in A$ there exists $a^{[p]} \in A$ such that

$$
a^{p}-\hbar^{p-1} a^{[p]} \in Z(A) .
$$

In particular $Z(A) \bmod \hbar$ contains $B^{p}$.

The existence of a weakly central quantization of a Poisson algebra $B$ imposes on it the following necessary condition: for any $b \in B$ there exists $b^{[p]} \in B$ such that

$$
\operatorname{ad}(b)^{p}=\operatorname{ad}\left(b^{[p]}\right)
$$

This motivates the following

2010 Mathematics Subject Classification. Primary 16S80; Secondary 17B63 . 
Definition 1.2. A Poisson $\mathbf{k}$-algebra $B$ is said to be a weakly restricted Poisson algebra if for any $f \in B$ there exists $f^{[p]} \in B$ such that

$$
\operatorname{ad}(f)^{p}=\operatorname{ad}\left(f^{[p]}\right) .
$$

Our goal is to study the local structure of a quantization algebra $A$ in terms of symplectic leaves of the affine Poisson variety $\operatorname{Spec} B$. Let us recall the definition of symplectic leaves in algebraic setting.

Definition 1.3. A symplectic leaf in an affine Poisson variety $X=\operatorname{Spec} B$ over $\mathbf{k}$ is a maximal locally closed connected subvariety $Y \subset X$ such that the corresponding ideal of its closure $I(\bar{Y})$ is a Poisson ideal and $Y$ is a smooth symplectic variety under the Poisson bracket induces from $B$.

By $\overline{W_{n, \hbar}}$ we will denote the complete Weyl algebra over $\mathbf{k}[[\hbar]]$ :

$$
\overline{W_{n, \hbar}}=\mathbf{k}[[\hbar]]\left\langle x_{1}, \cdots, x_{n}, y_{1}, \cdots, y_{n}\right\rangle, \quad\left[x_{i}, x_{j}\right]=0=\left[y_{i}, y_{j}\right],\left[x_{i}, y_{j}\right]=\hbar \delta_{i j}
$$

Similarly $W_{n}$ will denote the usual Weyl algebra

$$
W_{n}=\mathbf{k}\left[x_{1}, \cdots, x_{n}, y_{1}, \cdots, y_{n}\right], \quad\left[x_{i}, x_{j}\right]=0=\left[y_{i}, y_{j}\right],\left[x_{i}, y_{j}\right]=\delta_{i j}
$$

Let $A$ be a weakly central quantization of an affine Poisson variety $X=$ Spec $B$. Let $y \in \operatorname{Spec} B$ be a closed point, and $m_{y}$ be the corresponding maximal ideal. Denote by $A_{\bar{y}}$ the completion of $A$ with respect to two-sided ideal $\rho^{-1}\left(m_{y}\right)$-the pre-image of $m_{y}$ under the quotient $\rho: A \rightarrow A / \hbar A=B$. Put $\rho^{-1}\left(m_{y}\right) \cap Z(A)=Z_{y}$-an ideal in $Z(A)$ containing $\hbar$. Denote by $Z(A)_{\bar{y}}$ the completion of $Z(A)$ with respect to $Z_{y}$. Since $Z(A)$ is Noetherian and $A$ is a finitely generated $Z(A)$-module, it follows that $A_{\bar{y}}=A \otimes_{Z(A)} Z(A)_{\bar{y}}$. Also, $A_{\bar{y}}$ is a quantization of $B_{\bar{y}}$-the completion of $B$ with respect to $y$. Let $Y$ be a symplectic leaf in $\operatorname{Spec} B$ containing $y$. Let $\operatorname{dim} Y=2 n$. Our main result below (Theorem 1.4) shows that algebra $A_{\bar{y}}$ contains the completed Weyl algebra $\overline{W_{n, \hbar}}$. In what follows by a local Poisson $\mathbf{k}$-algebra we will mean a local k-algebra equipped with a Poisson bracket such that the maximal ideal is a Poisson ideal. More precisely, we have the following

Theorem 1.4. Algebra $A_{\bar{y}}$ is isomorphic to $\overline{W_{n, \hbar}} \otimes_{Z\left(\overline{W_{n, \hbar}}\right)} A_{\hat{y}}^{+}$, where $A_{\hat{y}}^{+}$is a weakly central quantization of a complete local Poisson algebra.

This result is motivated and may be seen as a positive characteristic analogue of results of Kaledin [ [K], Proposition 3.3] and Losev [L] on slice algebras of quantizations over $\mathbb{C}$.

\section{Auxiliary Lemmas}

We will recall few simple lemmas that will be used in the proof of our main result. We will include their proofs for the reader's convenience. 
Lemma 2.1. Let $S$ be a quantization of a Poisson algebra over $\mathbf{k}$, let $H \subset S$ be a $\mathbf{k}[[\hbar]]$-subalgebra which is isomorphic to a quotient of $\overline{W_{n, \hbar}}$. Assume that $Z(H) \subset Z(S)$. Let $S^{\prime}$ be the centralizer of $H$ in $S$. Then the multiplication map $H \otimes_{Z(H)} S^{\prime} \rightarrow S$ is an isomorphism. Moreover if $I \subset S$ is a two-sided ideal closed under $\frac{1}{\hbar} \operatorname{ad}(a), a \in S$, then $I=H \otimes_{Z(H)} I^{\prime}$, where $I^{\prime}=S^{\prime} \cap I$.

Proof. Proceeding by induction on $n$, it suffices to show the statement for $n=1$. Let $H$ be generated over $\mathbf{k}[[\hbar]]$ by elements $x, y$ where $[x, y]=\hbar$. By the assumption $x^{p}, y^{p} \in Z(S)$.

Let us view $S$ as a module over the Weyl algebra

$$
W_{1}(\mathbf{k})=\mathbf{k}\langle\alpha, D\rangle,[\alpha, D]=1,
$$

where the action by $\alpha$ is just the left multiplication by $x$, and $D(a)=$ $\frac{1}{\hbar}[y, a], a \in S$. Then $D^{p}(S)=0$. Therefore $S$ is a left $W_{1}(\mathbf{k}) /\left(D^{p}\right)$-module. On the other hand since $W_{1} /\left(D^{p}\right)$ is isomorphic to the $p \times p$ matrix algebra over $\mathbf{k}\left[\alpha^{p}\right]$, it follows that $S=k[x] \otimes_{\mathbf{k}\left[x^{p}\right]} S^{\prime}$, where $S^{\prime}$ is the centralizer of $y$ in $S$. Then $S^{\prime}$ is $\mathbf{k}[y]$-module closed under the bracket $\frac{1}{\hbar}[x,-]$. Arguing similarly as above we get that $S=H \otimes_{Z(H)} S_{1}$, where $S_{1}$ is the centralizer of $H$. Similarly $I=H \otimes_{Z(H)} I^{\prime}$, where $I^{\prime}=I \cap S^{\prime}$.

In the remainder of this section, given a ring $R$, we will denote by $x$ and $y$ the usual generators of the Weyl algebra over $R$ :

$$
W_{1}(R)=R\langle x, y\rangle, \quad[x, y]=1 .
$$

Lemma 2.2. Let $A$ be a weakly central quantization. Let $f, g \in A$ be such that $[f, g]=\hbar$. Then

$$
\left(f+g^{p-1} f^{[p]}\right)^{p}-\left(g^{p-1} f^{[p]}\right)^{p} \in Z(A) .
$$

Proof. Recall that in the Weyl algebra $W_{1}(R)$ over a commutative ring $R$ of characteristic $p$ the following identity (see for example [[B], Theorem 1.3])

$$
\left(x+r y^{p-1}\right)^{p}=x^{p}+\left(r y^{p-1}\right)^{p}-r, r \in R .
$$

In our case since $\left[\frac{1}{\hbar} f, g\right]=1$ and $f^{[p]}$ commutes with $f$ and $g$, by putting

$$
x=\frac{1}{\hbar} f, y=g, r=\frac{1}{\hbar} f^{[p]}
$$

in the above equality we obtain that

$$
\left(f+g^{p-1} f^{[p]}\right)^{p}-\left(g^{p-1} f^{[p]}\right)^{p}=f^{p}-\hbar^{p-1} f^{[p]} \in Z(A) .
$$

Lemma 2.3. Let $M$ be a left module over the Weyl algebra $W_{1}(\mathbf{k})$. Then

$$
M=x^{p-1} M+y M .
$$


Proof. In fact $W_{1}(\mathbf{k})=x^{i} W_{1}(\mathbf{k})+y W_{1}(\mathbf{k})$ for any $i \leq p-1$. This follows immediately from induction on $i$ since

$$
\left[x^{i}, \frac{1}{i} y\right]=x^{i-1}, 1 \leq i \leq p-1 .
$$

\section{The PROOF OF THE MAIN THEOREM}

We have the following key result.

Proposition 3.1. Let $(B, m)$ be a complete local Noetherian Poisson kalgebra with the maximal ideal $m$ such that $B$ is a finitely generated module over $B^{p}$. Let $I \subset m$ be a Poisson ideal. Let $A$ be a weakly central quantization of $B$. Assume that $S=B / I$ contains elements $x_{i}, y_{j}, 1 \leq i, j \leq n$ such that

$$
\left\{x_{i}, y_{j}\right\}=\delta_{i j}, \quad\left\{x_{i}, x_{j}\right\}=\left\{y_{i}, y_{j}\right\}=0, \quad \operatorname{ad}\left(x_{i}\right)^{p}=\operatorname{ad}\left(y_{j}\right)^{p}=0 .
$$

Then there are elements $z_{i}, w_{j} \in A, 1 \leq i, j \leq n$ such that they lift $x_{i}, y_{j}$ and

$$
\left[z_{i}, w_{j}\right]=\delta_{i j} \hbar, \quad\left[z_{i}, z_{j}\right]=\left[w_{i}, w_{j}\right]=0, \quad z_{i}^{p}, w_{j}^{p} \in Z(A) .
$$

Here is the Poisson version of this proposition without quantizations. Its proof is essentially the same as one of Proposition 3.1 .

Proposition 3.2. Let $(B, m)$ be a complete local weakly restricted Noetherian Poisson k-algebra with the maximal ideal $m$ such that $B$ is a finitely generated module over $B^{p}$. Let $I \subset m$ be a Poisson ideal. Assume that $S=B / I$ contains elements $x_{i}, y_{j}, 1 \leq i, j \leq n$ such that

$$
\left\{x_{i}, y_{j}\right\}=\delta_{i j}, \quad\left\{x_{i}, x_{j}\right\}=\left\{y_{i}, y_{j}\right\}=0, \quad \operatorname{ad}\left(x_{i}\right)^{p}=\operatorname{ad}\left(y_{j}\right)^{p}=0 .
$$

Then there are elements $z_{i}, w_{j} \in B, 1 \leq i, j \leq n$ such that they lift $x_{i}, y_{j}$ and

$$
\left\{z_{i}, w_{j}\right\}=\delta_{i j}, \quad\left\{z_{i}, z_{j}\right\}=\left\{w_{i}, w_{j}\right\}=0, \quad \operatorname{ad}\left(z_{i}\right)^{p}=\operatorname{ad}\left(w_{j}\right)^{p}=0 .
$$

Proof of Proposition 3.1. We will proceed by induction on $n$. Let $\rho: A \rightarrow$ $A / \hbar A=B$ denote the quotient map. Put $m^{\prime}=\rho^{-1}(m)$. Thus $m^{\prime}$ is the maximal ideal of $A$ and $A$ is complete in the $m^{\prime}$-adic topology. Put $m^{\prime[p]}=$ $\left\{x^{p}, x \in m^{\prime}\right\}$. Let us put $J^{\prime}=\rho^{-1}(I)$ and $J=J^{\prime}+m^{\prime[p]} A$. Then $J, J^{\prime}$ are two-sided ideals in $A$ and $\hbar \in J^{\prime} \subset J$. Moreover for any $a \in A$ we have

$$
\frac{1}{\hbar} \operatorname{ad}(a)\left(J^{\prime}\right) \subset J^{\prime}, \quad \frac{1}{\hbar} \operatorname{ad}(a)(J) \subset J .
$$

Let $f_{1}, g_{1} \in A$ be arbitrary lifts of $x_{1}, y_{1}$ respectively. Therefore

$$
\left[f_{1}, g_{1}\right]=\hbar+\hbar z_{1}, \quad z_{1} \in J^{\prime} .
$$

Now given elements $f_{n}, g_{n} \in A, n \geq 1$, such that

$$
x_{1}=f_{n} \bmod J^{\prime}, y_{1}=g_{n} \quad \bmod J^{\prime}, \quad\left[f_{n}, g_{n}\right]=\hbar+\hbar z_{n}, z_{n} \in J^{n},
$$

we will construct elements $f_{n+1}, g_{n+1} \in A$ such that

$$
\begin{gathered}
f_{n}=f_{n+1} \quad \bmod J^{\prime n}, \quad y_{1}=g_{n+1} \quad \bmod J^{\prime}, \\
{\left[f_{n+1}, g_{n+1}\right]=\hbar+\hbar z_{n+1}, \quad z_{n+1} \in J^{n+1} .}
\end{gathered}
$$


We claim that there exist $\omega \in J^{\prime n+1}, z_{n}^{\prime}, z_{n}^{\prime \prime} \in J^{\prime n}$ such that

$$
\hbar z_{n}=\hbar f_{n}^{p-1} z_{n}^{\prime}+\left[z_{n}^{\prime \prime}, g_{n}\right]+\hbar \omega .
$$

Indeed, notice that $J^{\prime n} / J^{\prime n+1}$ is a left module over the Weyl algebra

$$
W_{1}(\mathbf{k})=\mathbf{k}\langle x, y\rangle, \quad[x, y]=1,
$$

where $x$ acts as the multiplication by $f_{n}$, while $y$ acts as $-\frac{1}{\hbar} \operatorname{ad}\left(g_{n}\right)$. Now applying Lemma 2.3 to $J^{\prime n} / J^{\prime n+1}$ implies that

$$
z_{n} \in f_{n}^{p-1} J^{\prime n}+\frac{1}{\hbar}\left[J^{\prime n}, g_{n}\right]+J^{\prime n+1},
$$

which yields the desired result. Let us put $f_{n}^{\prime}=f_{n}-z_{n}^{\prime \prime}$. Then we may write

$$
\left[f_{n}^{\prime}, g_{n}\right]=\hbar+\hbar\left(f_{n}^{\prime}\right)^{p-1} w_{n}+\hbar \omega^{\prime}, \quad \omega^{\prime} \in J^{n+1}, \quad w_{n} \in J^{n} .
$$

Now recall that by our assumption on $A$, for any $a \in A$ there exists $a^{[p]} \in A$ such that

$$
\operatorname{ad}\left(a^{p}\right)=\operatorname{ad}(a)^{p}=\hbar^{p-1} \operatorname{ad}\left(a^{[p]}\right) .
$$

Thus, we have

$$
\left[f_{n}^{\prime}, \hbar^{p-1} g_{n}^{[p]}\right]=\operatorname{ad}\left(g_{n}\right)^{p-1}\left(\hbar\left(f_{n}^{\prime}\right)^{p-1} w_{n}+\hbar \omega^{\prime}\right)=(-1) \hbar^{p} w_{n}+\hbar^{p} f_{n}^{\prime} \omega_{n}^{\prime \prime}+\hbar^{p} \eta,
$$

for some $\omega_{n}^{\prime \prime} \in J^{n}, \eta \in J^{n+1}$. Then

$$
\left[f_{n}^{\prime}, g_{n}+\left(f_{n}^{\prime}\right)^{p-1} g_{n}^{[p]}\right]=\hbar+\hbar\left(f_{n}^{\prime}\right)^{p} \omega_{n}^{\prime \prime}+\hbar \eta^{\prime}, \quad\left(f_{n}^{\prime}\right)^{p} \omega_{n}^{\prime} \in J^{n+1}, \quad \eta^{\prime} \in J^{n+1} .
$$

Since $y_{1}=g_{n} \bmod J^{\prime}$ and $\operatorname{ad}\left(y_{1}\right)^{p}=0$, it follows that $g_{n}^{[p]} \in J^{\prime}$. Hence

$$
y_{1}=g_{n}+\left(f_{n}^{\prime}\right)^{p} g_{n}^{[p]} \bmod J^{\prime} .
$$

Therefore, we may put

$$
f_{n+1}=f_{n}^{\prime}, \quad g_{n+1}=g_{n}+\left(f_{n}^{\prime}\right)^{p-1} g_{n}^{[p]} .
$$

We see from the construction that the sequence $f_{n}, n \geq 1$ converges. Let us put $f=\lim f_{n}$. Thus we have that

$$
\left[f, g_{n}\right]=\hbar \bmod \hbar J^{n} .
$$

Writing $g_{n}=g_{1}+\hbar g_{n}^{\prime}$ and $\left[f, g_{1}\right]=\hbar+\hbar z_{1}$, we get that

$$
z_{1}=\left[f,-g_{n}^{\prime}\right] \bmod J^{n} \text {. }
$$

Hence

$$
z_{1} \in \bigcap_{n}[f, A]+J^{n}
$$

It follows from our assumptions that $A$ is a finitely generated module over a complete Noetherian ring $Z(A)$. Hence $[f, A]$ is finitely generated as a $Z(A)$-module. Then it follows from the Artin-Rees lemma that there exists $g^{\prime} \in A$ such that $\left[f, g^{\prime}\right]=z_{1}$. Putting $g=g_{1}-\hbar g^{\prime}$ we get that

$$
[f, g]=\hbar, \quad x_{1}=f \quad \bmod J^{\prime}, \quad y_{1}=g \quad \bmod J^{\prime} .
$$

Next we want to modify elements $f, g$ such that

$$
\operatorname{ad}(f)^{p}=\operatorname{ad}(g)^{p}=0, \quad[f, g]=\hbar .
$$


We will do so inductively: let $\phi_{n} \in A$ be such that

$$
\phi_{n} \quad \bmod J^{\prime}=x_{1}, \quad\left[\phi_{n}, g\right]=\hbar, \quad \phi_{n}^{[p]} \in m^{\prime n} .
$$

We have that by Lemma 2.2

$$
\left(\phi_{n}+g^{p-1} \phi_{n}^{[p]}\right)^{[p]}=\left(g^{p-1} \phi_{n}^{[p]}\right)^{[p]} .
$$

But $\left(g^{p-1} \phi_{n}^{[p]}\right)^{[p]} \in m^{\prime n+1}$. Indeed, this follows from the fact that if $x \in m^{n}$, then $x^{[p]} \in m^{n}$. Put $\phi_{n+1}=\phi_{n}+g^{p-1} \phi_{n}^{[p]}$. Then since $\phi_{n}^{[p]} \in J^{\prime}$, we get that $\phi_{n}=\phi_{n+1} \bmod m^{\prime n} J^{\prime}$ and $\phi_{n+1}^{[p]} \in m^{\prime n+1}$. Putting $\phi=\lim \phi_{n}$ we get that

$$
[\phi, g]=\hbar, \quad \phi^{[p]}=0 .
$$

Proceeding in the same manner with $g$ we get elements $z_{1}, w_{1} \in A$ such that

$$
\left[z_{1}, w_{1}\right]=\hbar, \quad x_{1}^{[p]}=0=y_{1}^{[p]}, \quad z_{1} \quad \bmod J^{\prime}=x_{1}, w_{1} \quad \bmod J^{\prime}=y_{1} .
$$

Let us put

$$
H=\mathbf{k}[[\hbar]]\left\langle z_{1}, w_{1}\right\rangle, \quad \overline{z_{1}}=z_{1} \quad \bmod \hbar, \quad \bar{w}_{1}=w_{1} \quad \bmod \hbar .
$$

Thus by Lemma 2.1 we have that $A=H \otimes_{Z(H)} A$, where $A_{1}$ is the centralizer of $H$ in $A$. It follows that $A_{1}$ is a weakly central quantization of the Poisson centralizer of $\overline{z_{1}}, \bar{w}_{1}$ in $B$, which we denote by $B_{1}$. Let $J_{1}^{\prime}=J^{\prime} \cap A_{1}$. Then by Lemma 2.1 we have that $J^{\prime}=H \otimes_{Z(H)} J_{1}^{\prime}$. In particular, $J_{1}^{\prime} \bmod \hbar=I^{\prime}$ is a Poisson ideal in $B_{1}$. It follows that $B_{1} / I^{\prime}$ contains $x_{i}, y_{j}, i, j \geq 2$. Proceeding by induction we are done.

Next we will recall the following characteristic $p$ analogue of the Darboux's theorem from [BK].

Lemma 3.3. Let $Y$ be an $2 n$-dimensional affine symplectic variety over $\mathbf{k}$ such that $\mathcal{O}(Y)$ is a weakly restricted Poisson algebra. Let $y \in Y$ be a closed point, and let $\mathcal{O}(Y)_{\bar{y}}$ denote the completion of the coordinate ring of $Y$ with respect to $y$. Then $\mathcal{O}(Y)_{\bar{y}}$ is isomorphic to the Poisson algebra $\mathbf{k}\left[\left[x_{1}, \cdots, x_{n}, y_{1}, \cdots, y_{n}\right]\right]$ with the bracket

$$
\left\{x_{i}, x_{j}\right\}=0=\left\{y_{i}, y_{j}\right\}, \quad\left\{x_{i}, y_{j}\right\}=\delta_{i j} .
$$

Proof. Let $m$ denote the maximal ideal of $\mathcal{O}(Y)_{\bar{y}}$. Denote by $m^{[p]}$ the ideal generated by $b^{p}, b \in m$. Then it follows from results of BezrukavnikovKaledin [[BK], Theorem 1.1, Proposition 3.4] that $\mathcal{O}(Y)_{\bar{y}} / m^{[p]}$ as a Poisson algebra is isomorphic to $\mathbf{k}\left[x_{1}, \cdots, x_{n}, y_{1}, \cdots, y_{n}\right] /\left(x_{1}^{p}, \cdots x_{n}^{p}, y_{1}^{p}, \cdots, y_{n}^{p}\right)$ with the Poisson bracket

$$
\left\{x_{i}, x_{j}\right\}=0=\left\{y_{i}, y_{j}\right\}, \quad\left\{x_{i}, y_{j}\right\}=\delta_{i j} .
$$

Now Proposition 3.2 yields the desired result. 
Proof of Theorem 1.4. Let $I$ be the Poisson ideal in $B_{\bar{y}}$ such that $B_{\bar{y}} / I=$ $\mathcal{O}(Y)_{\bar{y}^{-}}$the completion of $\mathcal{O}(Y)$ at $y$. It follows that $\mathcal{O}(Y)_{\bar{y}}$ is a weakly restricted Poisson algebra. Therefore by Lemma 1.4, it follows that

$$
\mathcal{O}(Y)_{\bar{y}}=\mathbf{k}\left[\left[x_{i}, y_{j}\right]\right], \quad 1 \leq i, j \leq n,
$$

with the usual Poisson bracket

$$
\left\{x_{i}, x_{j}\right\}=0=\left\{y_{i}, y_{j}\right\}, \quad\left\{x_{i}, y_{j}\right\}=\delta_{i j} .
$$

Now Proposition 3.1 and Lemma 2.1 imply that

$$
A_{\bar{y}}=\overline{W_{n, \hbar}} \otimes_{Z\left(\overline{W_{n, \hbar}}\right)} A_{\bar{y}}^{+},
$$

where $A_{\bar{y}}^{+}$is the centralizer of $\overline{W_{n, \hbar}}$ in $A_{\bar{y}}$, hence $\hbar \in A_{\bar{y}}^{+}$. Put $L=A_{\bar{y}}^{+} / \hbar A_{\bar{y}}^{+}$, hence $L \subset B_{\bar{y}}$. Then $A_{\bar{y}}^{+}$is a quantization of $L$ and

$$
B_{\bar{y}}=\widehat{\mathcal{O}(Y)_{\bar{y}}} \otimes_{{\widehat{\mathcal{O}(Y)_{\bar{y}}}}^{p}} L,
$$

where $\widehat{\mathcal{O}(Y)_{\bar{y}}}$ is a lift of $\mathcal{O}(Y)_{\bar{y}}$ under the quotient map $B_{\bar{y}} \rightarrow \mathcal{O}(Y)_{\bar{y}}$. Therefore $L$ is the Poisson centralizer of $\widehat{\mathcal{O}(Y)_{\bar{y}}}$ in $B_{\bar{y}}$. Put $m^{\prime}=\overline{m_{y}} \cap L$. Then $m^{\prime}$ is the maximal ideal of $L$. We claim that $m^{\prime}$ is a Poisson ideal. Indeed the image of $m^{\prime}$ in $B_{\bar{y}} / I=\mathcal{O}(Y)_{\bar{y}}$ is in the center, therefore

$$
\left\{m^{\prime}, m^{\prime}\right\} \subset I \cap L \subset m^{\prime} \text {. }
$$

Thus $A_{\bar{y}}^{+}$is a quantization of a complete local Poisson algebra $L$. Let $a \in A_{\bar{y}}^{+}$, then $a^{p}-\hbar^{p-1} a^{[p]} \in Z\left(A_{\bar{y}}\right) \subset Z\left(A_{\bar{y}}^{+}\right)$. It follows that $a^{[p]}$ is in the centralizer of $\overline{W_{n, \hbar}}$, hence $a^{[p]} \in A_{\bar{y}}^{+}$. Therefore $A_{\bar{y}}^{+}$is a weakly central quantization as desired.

As an immediate corollary of Theorem 1.4, one can reprove the following Kac-Weisfeiler type statement for quantizations as in [T].

Corollary 3.4. Let $M$ be an A-module which is finite and free over $\mathbf{k}[[\hbar]]$, such that $M / \hbar M$ is supported on $y$. Then $\operatorname{dim}_{\mathbf{k}[[\hbar]]} M$ is a multiple of $p^{n}$.

Proof. It follows that $M\left[\hbar^{-1}\right]$ is a nonzero module over $A_{\bar{y}}\left[\hbar^{-1}\right]$. Therefore, by Theorem $1.4 M\left[\hbar^{-1}\right]$ is a module over $\overline{W_{n}}\left[\hbar^{-1}\right]$. Hence $\operatorname{dim}_{\mathbf{k}[[\hbar]]} M$ is divisible by $p^{n}$.

Acknowledgements. I am very grateful to the referee for many useful suggestions that led to the improvement of the paper.

\section{REFERENCES}

[B] V. Bavula, The group of automorphisms of the first Weyl algebra in prime characteristic and the restriction map, Glasg. Math. J. 51 (2009), no. 2, 263-274.

[BK] R . Bezrukavnikov, D. Kaledin, Fedosov Quantization in positive characteristic, J. Amer. Math. Soc. 21 (2008), no. 2, 409-438.

[L] I. Losev, Appendix to Poisson traces and D-modules on Poisson varieties, P. Etingof,

T. Schedler, Geom. Funct. Anal. 20 (2010), no. 4, 958-987. 
[K] D. Kaledin, Symplectic singularities from the Poisson point of view, J. Reine Angew. Math. 600 (2006), 135-156.

[T] A. Tikaradze, An analogue of the Kac-Weisfeiler conjecture, J. Algebra 383 (2013), 168-177.

Department of Mathematics, University of Toledo, Toledo, Ohio 43606

E-mail address: tikar06@gmail.com 